Muñoz-San Roque, I., Martín-Alonso, J. F., Prieto-Navarro, L. y Urosa-Sanz, B. (2016). Autopercepción del nivel de desarrollo de la competencia de aprender a aprender en el contexto universitario: propuesta de un instrumento de evaluación. Revista de Investigación Educativa, 34(2), 369-383.

DOI: http://dx.doi.org/10.6018/rie.34.2.235881

\title{
Autopercepción del nivel de desarrollo de la competencia de Aprender a Aprender en el contexto universitario: propuesta de un instrumento de evaluación
}

\author{
Self-perceived level of development of learning to learn \\ competence in the university context: \\ a proposed measuring instrument
}

Isabel Muñoz-San Roque, José Francisco Martín-Alonso, Leonor Prieto-Navarro y Belén Urosa-Sanz Departamento de Educación, Métodos de Investigación y Evaluación. Universidad Pontificia Comillas de Madrid

\begin{abstract}
Resumen
Este trabajo se enmarca en el Proyecto de Investigación de la Universidad Pontificia Comillas denominado EDUCOMPET. El artículo presenta el proceso de diseño y de validación de una escala para medir la autopercepción del nivel de desarrollo de la competencia de aprender a aprender en estudiantes universitarios. La integración de las competencias clave a nivel europeo requiere de una fundamentación teórica sólida que sustente la adecuación de los instrumentos para su medida. En este trabajo se propone una estructura del constructo desde la perspectiva de la autorregulación. La estructura se centra en tres dimensiones: gestión del aprendizaje, autoevaluación del proceso de aprendizaje y autoconocimiento como aprendiz. Se ha aplicado el instrumento a una muestra de 458 estudiantes universitarios. Se realizaron análisis exploratorios y confirmatorios para validar el modelo teórico del que se partía y se obtuvo una fiabilidad e indices de ajuste adecuados para justificar la validez del instrumento.

Palabras clave: competencia de aprender a aprender; autorregulación; evaluación de competencias.

Correspondencia: Isabel Muñoz-San Roque. E-mail: isabelmsanroque@comillas.edu. José Francisco Martín Alonso. E-mail: jfmartin@comillas.edu. Belén Urosa Sanz. E-mail: burosa@comillas.edu. Leonor Prieto Navarro. E-mail: lprieto@comillas.edu. Departamento de Educación, Métodos de Investigación y Evaluación, Universidad Pontificia Comillas de Madrid, C/ Universidad Comillas, 3, 28049, Madrid (España).
\end{abstract}




\begin{abstract}
This paper is part of a research project at the Universidad Pontificia Comillas de Madrid called EDUCOMPET. This article describes the process involved in designing and validating a scale to measure the self-perception among college students of the development of the learning to learn competence. Key competences are one of the most important facts in European education politics and require a solid theoretical foundation that supports the evaluation instruments. In this paper, the structure of the construct comes from the theory of self-regulation. The structure focuses on three dimensions: learning management, self-assessment and self-learning process as an apprentice. The instrument has been applied to a sample of 458 college students. Exploratory and confirmatory analysis were conducted to validate the theoretical model and reliability indices, and the suitability of the structural model was determined to justify the validity of the instrument.

Keywords: learning to learn; self-regulation; skills assessment.
\end{abstract}

\title{
Introducción
}

Las competencias clave propuestas por la Unión Europea (Unión Europea, 2007) suponen una de las políticas educativas más importantes en el ámbito educativo europeo por dos motivos (Leat, Thomas \& Reid, 2012; Valle \& Manso, 2013): porque el paradigma de las competencias clave ha tenido repercusiones reales en las políticas nacionales de todos los Estados miembros, y en segundo lugar, porque con este paradigma se quiere responder de forma más adecuada a los retos propios de la Sociedad del Conocimiento.

Además, el enfoque centrado en las Competencias Clave integra un concepto fundamental en la política educativa europea como es el del Aprendizaje Permanente que aparece por primera vez en 1995 en el Libro Blanco Teaching and Learning. Towards a Learning Society, Comisión Europea (1995). En este momento se empiezan iniciativas importantes como el programa LifeLong learning 2007-2013, el plan de acción para el aprendizaje de adultos y las competencias clave para el aprendizaje permanente, como indican Valle y Manso (2013).

\section{La competencia de aprender a aprender en el contexto europeo}

En 1997 se publica el documento del Consejo de Europa en el que se recogen las conclusiones de Berna donde se hace alusión a la competencia de aprender a aprender como uno de los cambios relevantes que tienen que producirse para incorporar las competencias a la escuela.

Se ha considerado el Consejo de Europa celebrado en Lisboa en el año 2000 como el inicio de la búsqueda de indicadores para el estudio de las competencias clave, entre ellas la de aprender a aprender.

El Consejo Europeo de Estocolmo (2001), aprobó tres objetivos y 13 sub-objetivos estratégicos para los sistemas educativos de los países miembros. Ya en el objetivo 1.2 se señala aprender a aprender entre una preselección inicial de competencias clave. 
Un año después, el Consejo Europeo de Barcelona (2002) aprobó un programa de trabajo Educación y Formación 2010 para conseguir estos objetivos. En él se destaca la competencia de aprender a aprender como un elemento clave tanto para sistemas educativos y de formación, como para que los grupos de trabajo permanentes establezcan indicadores.

El siguiente paso fue formar una serie de grupos de trabajo de los cuales uno se centró en las competencias clave. De este modo se presenta a la Comisión Europea el documento Propuesta de recomendación del parlamento europeo y del consejo sobre las competencias clave para el aprendizaje permanente (Education Council, 2006).

La necesidad de establecer indicadores de medición de la competencia de aprender a aprender tuvo un empuje fundamental con la creación del CRELL (Centre for Research on Lifelong Learning) en el año 2005, que junto con la Red Europea de Responsables de Políticas para la Evaluación del Sistema Educativo se propuso desarrollar un instrumento para medir la competencia. Para ello, además del análisis conceptual, consideraron cuatro instrumentos de evaluación (Hoskins \& Fredriksson, 2008):

- Las pruebas de aprender a aprender, de la Universidad de Helsinki dentro del proyecto "La vida como aprendizaje" (LEARN), (Hautamäki et al., 2002).

- El Inventario de aprendizaje permanente y eficaz (Effective Lifelong Learning Inventory-ELLI), de la Universidad de Bristol (Deakin-Crick, Broadfoot, \& Claxton, 2004).

- La prueba de competencias transversales desarrolladas por la Universidad de Amsterdam (cross-curricular skills test-CCST) (Elshout-Mohr, Meijer, Oostdam, \& Van Gelderen, 2004).

- La prueba de Metacognición desarrollada por la Universidad Autónoma de Madrid (Moreno, 2002).

Desde estos estudios se definió la competencia y se desarrolló una prueba pre-piloto de medición (Kupiainen, Hautamakï, \& Rantanen, 2008) que se aplicó en varios países, entre ellos España (Moreno, Cercadillo, \& Martínez, 2008). De este modo se definieron tres grandes dimensiones: Afectiva, Cognitiva y Metacognitiva.

Aunque los autores expresaron su confianza en la capacidad del informe para ayudar a abrir una nueva etapa en el debate sobre la búsqueda de un indicador europeo común de aprender a aprender (Kupiainen et al., 2008), los que analizaron los resultados de la muestra española (Moreno et al., 2008) expresaron, aparte de la conveniencia de contar con una muestra más grande, la necesidad de una mayor profundización en el análisis teórico de aprender a aprender que justificara mejor la validez del constructo.

Posteriormente, diversos autores españoles han definido el constructo de la competencia de aprender a aprender desde diversas perspectivas para poder llegar a construir mecanismos que permitan su evaluación (Carretero \& Fuentes, 2010; Jornet-Meliá, García-Bellido, \& González-Such, 2012; Martín \& Moreno, 2007; Villardón-Gallego, Yániz, Achurra, Iraurgi, \& Aguilar, 2013, entre otros). 


\section{La competencia de aprender a aprender desde la perspectiva de la autorregulación}

Para definir la estructura de la competencia de aprender a aprender en este trabajo se parte de la perspectiva de la autorregulación (siguiendo a Bandura, 1991; Meyer \& Turner, 2002; Pekrun, Goetz, Titz, \& Perry, 2002; Zimmerman, 1989, 1990, 1995, 2013 etc.). Según Zimmerman (1989) una persona autorregulada se caracteriza por ser un participante activo en sus procesos personales de aprendizaje en lo cognitivo, motivacional y conductual. En 2002 este mismo autor lo define como un conjunto de procesos específicos que incluyen la definición de objetivos próximos para uno mismo, la adopción de estrategias adecuadas, la monitorización selectiva del progreso personal, la reestructuración del ambiente físico y social para hacerlo compatible con los propios objetivos, el control efectivo del tiempo, la autoevaluación de los métodos utilizados, la atribución interna de los efectos y la adopción de métodos futuros.

Actualmente los modelos teóricos derivados de la corriente sociocognitiva son los que han demostrado una aproximación más profunda y detallada del proceso y de los componentes implicados en la autorregulación (Salmerón \& Gutiérrez, 2012). Este enfoque también es defendido por el propio Zimmerman (2013) que habla de los componentes personales, conductuales y ambientales, evidenciando el gran aporte que realizó Bandura (1986).

El objetivo central de esta investigación es proponer un instrumento de medición para medir la Autopercepción del nivel de desarrollo de la competencia de aprender a aprender.

La estructura de la escala se fundamenta en primer lugar en los modelos teóricos de varios autores que miden la autorregulación: el modelo integrador propuesto por Torre (2007) que sugiere cuatro dimensiones de la autorregulación académica: la contextual, la cognitiva, la conductual y la emocional y en cada dimensión se plantean dos indicadores, uno para referirse al conocimiento y otro para referirse al control o gestión de cada una de las dimensiones; las estrategias de autorregulación del aprendizaje propuestas por Zimmerman (2013); los subprocesos de la autorregulación de Bandura (1986) que denomina auto-observación, auto-evaluación e influencias autorreactivas; y Pintrich, Smith, García y Mckeachie (1991) cuando miden autorregulación académica. También se tienen en cuenta las dimensiones utilizadas por otros autores como Hautamaki et al. (2002), Deakin-Crick et al. (2004), Martín \& Moreno (2007), Martínez-Fernández (2007), siguiendo a O’Neil y Abedi (1996), Hoskins y Fredriksson (2008), Bolívar (2009), Jornet-Meliá, García-Bellido y González Such (2012), Villardón-Gallego et al. (2013), cuando definen y miden la competencia de aprender a aprender.

Partiendo del análisis de los modelos teóricos de los autores antes mencionados, modelos que les permitieron construir instrumentos de medida, se realizó una selección de aquellas dimensiones que eran comunes a todos ellos y que definían la percepción de la competencia de aprender a aprender desde la perspectiva de la autorregulación. De esta selección surge una estructura teórica basada en tres dimensiones: Gestión del proceso de aprendizaje, Autoevaluación del proceso y Autoconocimiento como aprendiz. A continuación se justifica cada dimensión partiendo de los autores antes mencionados. 


\section{Gestión del proceso de aprendizaje}

La dimensión de Gestión del aprendizaje incluye los siguientes aspectos: Planificar y gestionar cognitivamente el proceso que va siguiendo el estudiante para optimizarlo, planteándose objetivos realistas, Zimmerman (2013) integra este aspecto en una dimensión que denomina Goal-setting and planning, Martínez-Fernández (2007) integra esta dimensión para medir estrategias metacognitivas y VillardónGallego et al. (2013) plantean la importancia de que el aprendiz persevere para conseguir los objetivos planteados. En el plano más conductual es importante que el estudiante ejecute las acciones más adecuadas para desarrollar eficientemente una determinada tarea, Zimmerman (2013) lo incluye en varias dimensiones como son Organizing and transforming, Seeeking information, Keeping records and monitoring y Villardón-Gallego et al. (2013) aluden a este aspecto a través del uso de diferentes estrategias adaptadas a las tareas requeridas. Pintrich y De Groot (1990) ya sugerían la planificación, el manejo de estrategias y el control del esfuerzo en un aprendizaje autorregulado.

\section{Autoevaluación del proceso}

Es fundamental, desde la perspectiva de la autorregulación la autoevaluación que realiza el estudiante del proceso que está siguiendo cuando aprende para poder mejorarlo. Zimmerman (2013) lo asume como una dimensión central de las estrategias de autorregulación a la que denomina Self-evaluation y en este sentido sugiere la importancia de que el estudiante realice una comprobación del proceso que está siguiendo mientras aprende. Hautamaki et al. (2002) también tienen en cuenta esta dimensión cuando definen la competencia de aprender a aprender y Martínez-Fernández (2007) lo incluye en las estrategias metacognitivas.

\section{Autoconocimiento como aprendiz}

En la vertiente cognitiva se incluye si el estudiante es consciente del grado de conocimientos que tiene y de su manera particular de procesar la información y es capaz de identificar sus hábitos de estudio, coincidiendo con Villardón-Gallego et al. (2013) que lo integran en una dimensión que denominan conocimiento personal como aprendiz, Hautakami et al. (2002) hablan de la autopercepción del aprendizaje académico en este mismo sentido y Deakin-Crik et al. (2004) lo denominan conocimiento estratégico entendiéndolo como ser consciente del propio proceso de aprendizaje en pensamientos, sentimientos y acciones. Martínez-Fernández (2007) lo incluye en sus estrategias metacognitivas en un indicador que mide si el estudiante es consciente de los procesos de pensamiento que utiliza al aprender.

Y, por último, desde la vertiente emocional se tiene en cuenta si el estudiante es consciente de sus fuerzas y debilidades a la hora de aprender, en consonancia con Villardón-Gallego et al. (2013) que incluyen este aspecto en su herramienta de medida y con Martín y Moreno (2007) que aluden a la autoconciencia emocional. 


\section{Método}

\section{Participantes}

La selección de la muestra es incidental y se obtuvo cumpliendo con tres requisitos fundamentales, debían ser alumnos tanto de Universidades públicas, como privadas de la Comunidad de Madrid, pertenecer a las diferentes áreas de conocimiento y estar matriculados en diferentes cursos. La muestra respondiente está formada por 458 sujetos, 331 son mujeres y 127 son hombres. 187 son del área de Ciencias Sociales y de la Salud (Psicología, Magisterio, Trabajo Social y Educación Social), 66 de Ingeniería y estadística aplicada, 85 de Traducción e Interpretación, Relaciones Internacionales y periodismo, 120 estudian Administración y Dirección de Empresas, Economía y Comercio. 135 alumnos estudian en Universidades públicas (29,5\%) y 323 en Universidades privadas $(70,5 \%)$. En cuanto a los cursos, $18 \%$ son de $1^{\circ}, 24,1 \%$ de $2^{\circ}, 30 \%$ de $3^{\circ}, 25,9$ de $4^{\circ}, 2 \%$ de $5^{\circ}$ o estudios finalizados. La edad media de la muestra es de 21,62 años y una desviación típica de 4,07.

\section{Instrumentos}

Se utilizó un cuestionario en el que se incluían:

- Datos sociodemográficos (Estudios que están realizando, Curso, Universidad, Sexo, Edad, № de suspensos en la última evaluación, Nota media aproximada en el último parcial)

- Escala de Autopercepción del nivel de Desarrollo de la Competencia de Aprender a Aprender (EADCAA)

Para diseñar la escala se ha partido del modelo teórico planteado por Torre (2007) que presenta una estructura del constructo de Autorregulación basada en cuatro dimensiones (contexto, cognición, conducta y emoción) y en cada dimensión utiliza dos subdimensiones (conocimiento y control/gestión) y también se ha partido de las estrategias de autorregulación del aprendizaje propuestas por Zimmerman (2013), el enfoque desarrollado por Pintrich et al. (1991) y las dimensiones aportadas en varios instrumentos de medida como los de Hautamäki et al. (2002), Moreno (2002), DeakinCrick et al. (2004), Elshout-Mohr et al. (2004) y Martínez-Fernández et al. (2009).

En primer lugar, se redactaron 19 ítems. Para la selección de los ítems definitivos se siguieron dos criterios fundamentales:

- Validación de contenido a través de la valoración de la pertinencia y adecuación de las dimensiones propuestas y de los ítems por parte de cuatro profesores universitarios expertos en el área de la psicología de la Educación. Para ello se utilizó el modelo CIRA de Hernández-Franco y Gonzalo-Misol (2009) y se eliminaron aquellos ítems cuya pertinencia y adecuación para medir la percepción de la competencia fuese baja. 
- Análisis del funcionamiento psicométrico de los ítems. Se mantuvieron aquellos ítems cuya mayor saturación factorial en la matriz rotada estuviera en la dimensión que postulaba el modelo teórico planteado y que no tuvieran pesos superiores a .30 en el resto de factores.

Tras estos análisis se decidió finalmente mantener 9 ítems cuyas opciones de respuesta mostraban el nivel de desarrollo percibido de los estudiantes en una escala de 1 a 6 (poco desarrollado a muy desarrollado) y que mantenían los diferentes matices conceptuales de los que partíamos desde la base teórica.

En el cuestionario también se añadieron dos instrumentos para poder analizar la validez criterial de la Escala.

- Escalas de Uso de los Enfoques de aprendizaje Profundo y Superficial SRPQ (Biggs, Kember, \& Leung, 2001, traducida por Torre, 2007)

- Escala de Autoeficacia académica (Torre, 2007)

\section{Procedimiento}

Se diseñó una batería de pruebas on-line en la que se incluía, además del cuestionario de datos sociodemográficos, la Escala de Autopercepción del nivel de Desarrollo de la Competencia de Aprender a Aprender (EADCAA), las Escalas para medir los Enfoques de aprendizaje Profundo y Superficial SRPQ (Biggs, Kember, \& Leung, 2001, traducida por Torre, 2007) y la Escala de Autoeficacia académica (Torre, 2007), este procedimiento permitió recoger toda la información necesaria para la validación de los instrumentos y la verificación de hipótesis al mismo tiempo, sin introducir nuevas fuentes de variación no controlada. Se pidió a profesorado universitario de diferentes áreas de conocimiento y de universidades públicas y privadas madrileñas que enviara el enlace del cuestionario explicando la naturaleza y función de la investigación a sus alumnos para que lo completaran, garantizando su confidencialidad y voluntariedad. Se recogieron los datos entre enero y marzo del 2014.

\section{Resultados}

Se calcularon los coeficientes de consistencia interna a través del alfa de Cronbach y los índices de homogeneidad. Se analizó la validez de constructo a través de un análisis factorial exploratorio (análisis de componentes principales y rotación Promax), se verificó la adecuación muestral de la escala utilizando la prueba de Kaiser-MeyerOlkin y el test de esfericidad de Bartlett. Se comprobó la validez criterial calculando la correlación de la escala y sus factores con variables con las que la teoría indica tener relación (Enfoques de Aprendizaje Profundo y Superficial: SRPQ Biggs et al., 2001, traducida por Torre, 2007; Percepción de Autoeficacia, Torre, 2007).

Para realizar el análisis factorial confirmatorio se utilizaron técnicas estructurales de covarianza utilizando el programa EQS (Bentler, 1995), para la estimación de parámetros se utilizó el método de máxima verosimilitud y para la bondad de ajuste del modelo la prueba Ji2, por la sensibilidad de esta prueba a las variaciones del tamaño muestral 
se empleó la raíz cuadrada de la media de los residuos estandarizados (Standarized Root Mean Square Residual- SRMR), el error de aproximación cuadrático medio (Root Mean Square Error of Aproximation-RMSEA) y su intervalo de confianza del 90\%.

Se mostrará el modelo final con los parámetros de las relaciones estructurales indicando los coeficientes factoriales estandarizados y los errores de estimación.

Tabla 1

Estadísticos descriptivos y análisis de consistencia interna de la escala que mide nivel de desarrollo percibido de la competencia de aprender a aprender

\begin{tabular}{|c|c|c|c|c|}
\hline ENUNCIADO DE LOS ÍTEMS & Media & $\begin{array}{c}\text { Desv. } \\
\text { típ. }\end{array}$ & $r$ & Alfa \\
\hline
\end{tabular}

1. Soy capaz de tener cierto control sobre cómo aprendo (por ejemplo. fijarme objetivos. detectar lo que no funciona cuando estoy estudiando y modificar el proceso para mejorarlo)

$4.47 \quad 1.15 \quad .72$

2. Organizo mi estudio planteándome objetivos realistas (antes de empezar soy capaz de organizar el tiempo de estudio necesario para conseguir los objetivos que me he planteado)

3. Establezco tiempos para el estudio

$\begin{array}{lll}4.22 & 1.31 \quad .64\end{array}$

4. Soy capaz de utilizar diferentes estrategias de aprendizaje en función de la tarea requerida

$4.21 \quad 1.37 \quad .60$

$\begin{array}{lll}4.30 & 1.25 \quad .66\end{array}$

Autoevaluación del proceso

5. Comprobar durante la tarea si ésta está respondiendo a los objetivos que me he planteado

$3.99 \quad 1.21 \quad .69$

6. Comprobar si los resultados se corresponden a los objetivos que me planteé al iniciar la tarea

$4.23 \quad 1.15 \quad .64$

\begin{tabular}{llll}
\hline Autoconocimiento como aprendiz & & & \\
\hline $\begin{array}{l}\text { 7. Soy consciente de mi grado de conocimientos y de mi } \\
\text { manera particular de procesar la información }\end{array}$ & 4.87 & 0.96 & .57 \\
$\begin{array}{l}\text { 8. Soy capaz de identificar mis hábitos de estudio y puedo } \\
\text { describirlos adecuadamente }\end{array}$ & 4.59 & 1.13 & .66 \\
$\begin{array}{l}\text { 9. Soy consciente de mis virtudes y dificultades cuando estoy } \\
\text { estudiando o aprendiendo }\end{array}$ & 4.86 & 0.99 & .56 \\
\hline $\begin{array}{l}\text { Nivel de desarrollo percibido Competencia de Aprender a } \\
\text { Aprender (Total) }\end{array}$ & 4.43 & 0.84 & \\
$\begin{array}{l}\text { Gestión del aprendizaje } \\
\text { Autoevaluación del proceso }\end{array}$ & 4.31 & 1.04 & \\
Autoconocimiento como aprendiz & 4.13 & 1.11 & .83 \\
\hline
\end{tabular}

En la tabla 1 pueden verse las medias y desviaciones típicas de los ítems y de las dimensiones, los índices de homogeneidad de los ítems y el alfa de Cronbach de la escala y de sus dimensiones. La fiabilidad de la escala con los 9 ítems a través del 
alfa de Cronbach es de .88 , por tanto muy alta y nos permite concluir que la escala discrimina adecuadamente a los estudiantes en la percepción de dominio de la competencia de aprender a aprender. Si analizamos los índices de homogeneidad de los ítems (correlación de cada ítem con el total de la escala sin el ítem), observamos que todos tienen valores superiores a .51 .

La prueba KMO (=.859) y el test de esfericidad de Bartlett (Ji2=1935.75; $\mathrm{p}<.001)$ nos indican que la muestra es adecuada para llevar a cabo la factorización de los ítems. Se realizó el Análisis factorial Exploratorio a través de componentes principales y rotación Promax y se extraen tres factores a través del criterio de Kaiser, que coinciden con el modelo teórico planteado en el Análisis Factorial Confirmatorio. Estos tres factores explican un $73 \%$ de la varianza total.

Para la validez de criterio, se calculó la correlación de la escala con la percepción de uso de los enfoques de aprendizaje superficial y profundo (SRPQ) Escalas de Biggs et al., (2001), traducción de Torre (2007), la percepción de Autoeficacia, escala elaborada por Torre (2007) y con el rendimiento académico (debían indicar la nota media obtenida en el último parcial). Se obtuvieron correlaciones de $\mathrm{r}=.48(\mathrm{p}<.001)$ con el uso de enfoque profundo, $\mathrm{r}=-.31(\mathrm{p}<.001)$ con el enfoque superficial y $\mathrm{r}=.53(\mathrm{p}<.001)$ con el nivel de Autoeficacia. La relación de la percepción del nivel de desarrollo de la competencia de aprender a aprender con el rendimiento obtenido es de $r=.32(p<.001)$.

Para llevar a cabo el análisis confirmatorio se probaron tres modelos estructurales: modelo de un único factor, modelo de tres factores relacionados y modelo de tres dimensiones subsumidas en un factor de segundo orden. Los índices de bondad de ajuste de los tres modelos se presentan en la tabla 2 y el diagrama del modelo de tres factores relacionados se presenta en el gráfico 1.

Tabla 2

Modelos estructurales de lcs basados en análisis factorial confirmatorio ( $N=458)$. Estimadores robustos de máxima verosimilitud.

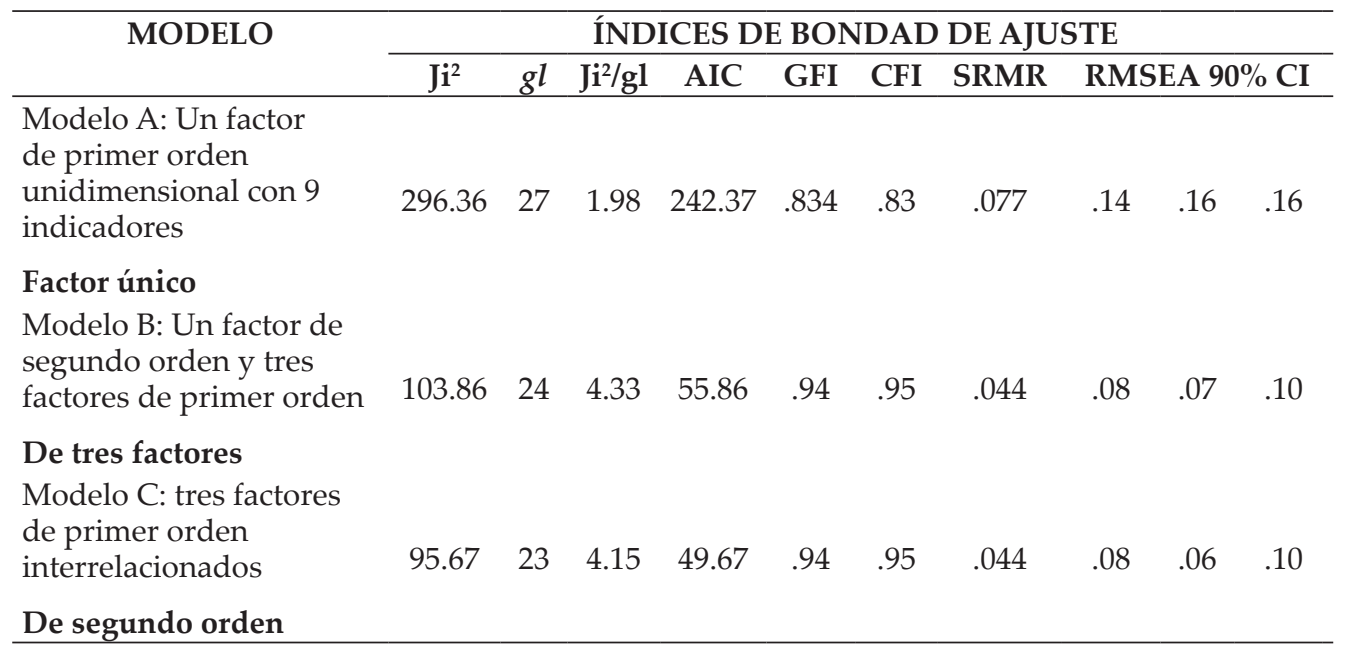


Teniendo en cuenta que valores de RMSEA $\leq .06$ son considerados como indicadores de un aceptable grado de ajuste (Hu \& Bentler, 1999), además un CFI en torno a .95 y un RMSEA por debajo de .08 son también considerados generalmente como buenos indicadores del ajuste entre el modelo y los datos (Savalei \& Bentler, 2006). En este trabajo el análisis de los índices de ajuste nos permite comprobar que CFI tiene un valor de .95 que podemos considerar medio-bajo y un RMSEA igual a .08 (90\% CI RMSEA. = .07 - .10). Por tanto, podemos concluir que a pesar de la significatividad del Ji2, sin embargo y como puede apreciarse en el diagrama 1, todos los índices calculados en el modelo de tres factores relacionados nos ofrecen un buen ajuste entre el modelo teórico postulado y los datos y podemos considerar, por tanto, el modelo aceptable, existiendo un grado de congruencia satisfactoria entre el modelo teórico hipotetizado y lo que muestran los datos empíricos de nuestra muestra.

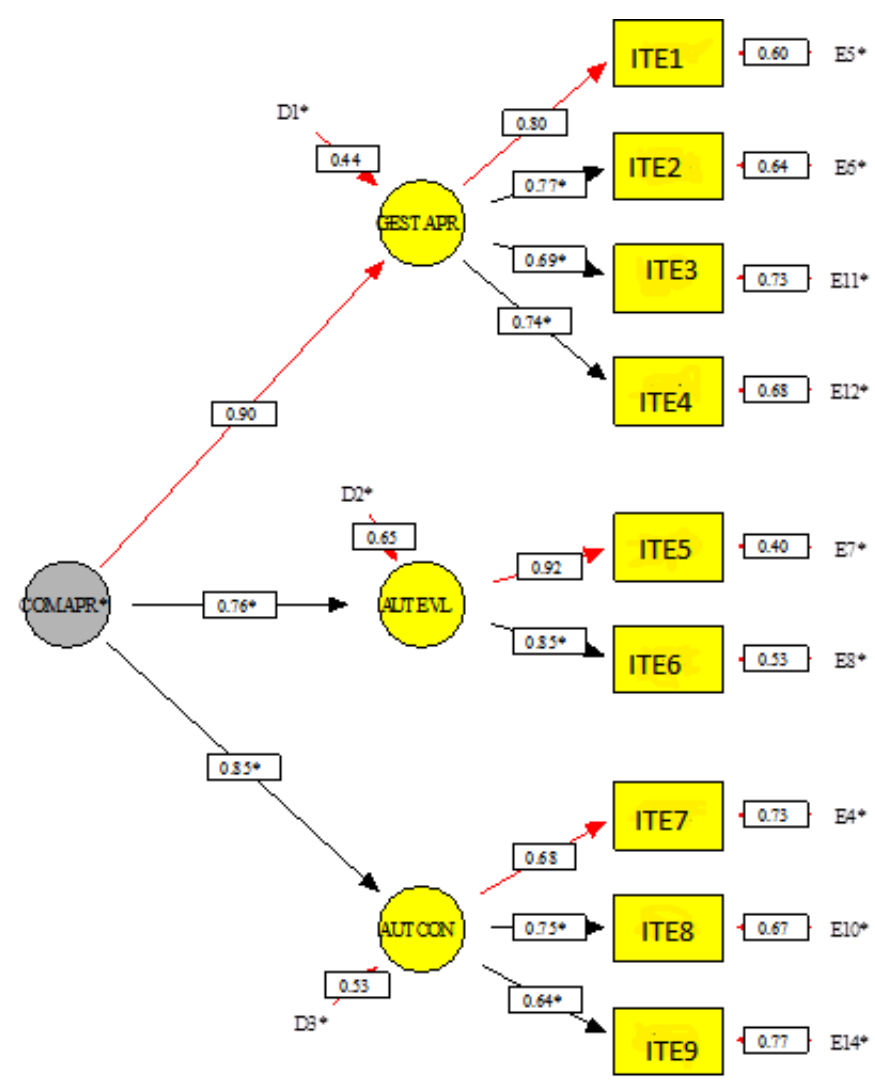

Modelo B: Modelo de tres factores

$\mathrm{Ji}^{2}=103,86 ; \mathrm{gl}=24 ; \mathrm{Ji2} / \mathrm{gl}=4,33 ; \mathrm{AIC}=55,86 ; \mathrm{GFI}=.94 ; \mathrm{CFI}=.95 ; \mathrm{SRMR}=.044 ; \mathrm{RMSEA}=.08$ (.07-.10)

Figura 1. Diagrama del modelo de tres factores relacionados 


\section{Discusión}

El objetivo central de esta investigación es proponer un instrumento de evaluación para medir la Autopercepción del nivel de desarrollo de la competencia de aprender a aprender. Podemos afirmar que el modelo propuesto cumple con los requisitos necesarios de fiabilidad y validez al obtener un índice alfa de Cronbach de .88 y unos índices de bondad de ajuste en el análisis confirmatorio que se consideran adecuados.

Se puede aportar también evidencia de validez criterial, ya que la correlación del nivel de desarrollo percibido de la competencia y los niveles de uso de un enfoque de aprendizaje profundo y superficial, los niveles de autoeficacia y el rendimiento académico, correlaciones que son coherentes y de magnitud parecida a lo encontrado en investigaciones previas sobre el tema como las de Torre (2007), García-Ros y PérezGonzález (2011), Salmerón H., Gutiérrez, Salmerón P. y Rodríguez (2011), Muñoz-San Roque, Prieto-Navarro y Torre Puente (2012), Muñoz-San Roque \& Martínez-Felipe (2012), Gutiérrez-Braojos, Salmerón-Pérez, \& Muñoz-Cantero (2014), López-Aguado \& Gutiérrez-Provecho (2014), entre otros.

La estructura propuesta en tres dimensiones nos permite definir el nivel de desarrollo percibido de la competencia de aprender a aprender desde la Gestión del proceso de Aprendizaje, la Autoevaluación del proceso y el Autoconocimiento como aprendiz. Esta estructura es coherente con la visión planteada por Pintrich et al. (1991), Hautamaki et al. (2002), Deakin-Crik et al. (2004), Martín \& Moreno (2007), Martínez-Fernández (2007), Zimmerman (2013) y Villardón-Gallego et al. (2013), entre otros.

Al analizar los estadísticos descriptivos (tabla 1) de los ítems observamos que todos tienen, en una escala de 1 a 6, una media superior a 3.9. Lo que perciben tener menos desarrollado es la autoevaluación del proceso de aprendizaje, aunque con una media que puede considerarse más bien alta en una escala de 1 a 6 (media $=4.13$, desv. tip.=1.11). Las diferencias entre las tres medias (a través de ANOVA medidas repetidas) son estadísticamente significativas $(\mathrm{p}<.001)$ y si analizamos las combinaciones de pares de medias (a través del DHS de Tukey) también son estadísticamente significativas.

Se realizó también un análisis de regresión por pasos sucesivos para comprobar cómo afectaban variables como Autopercepción del nivel de desarrollo de la competencia de aprender a aprender, uso de Enfoques de Aprendizaje Profundo y Superficial y el nivel de Autoeficacia percibida sobre el rendimiento académico. Encontramos que el mayor efecto predictivo es del nivel de autoeficacia, en segundo lugar la del uso de un Enfoque Profundo de Aprendizaje y en tercer lugar, de la Autopercepción del nivel de desarrollo de la competencia de aprender a aprender. El nivel de uso superficial no predice significativamente la variable rendimiento. García-Ros y Pérez-González (2011), Broc-Cavero (2011) y Dávila, García-Artiles, Pérez-Sánchez y Gómez-Déniz (2015) encuentran datos parecidos cuando analizan el valor predictivo de las habilidades de autorregulación y otras variables relacionadas con el aprendizaje sobre el rendimiento académico.

Partiendo del análisis del perfil de los estudiantes de las diferentes áreas de conocimiento analizadas a través de ANOVA de un factor y contrastes posteriores de Scheffé, comprobamos que los estudiantes de Economía y Finanzas perciben tener niveles menores de autoeficacia, de uso de un enfoque de aprendizaje profundo y de desarrollo de 
la competencia de aprender a aprender, sin embargo, tienen niveles mayores de uso de un enfoque superficial, sobre todo si les comparamos con el área de Ciencias Sociales y de la Salud y de Traducción y Relaciones Internacionales y estudios Lingüísticos. Estos resultados coinciden con los encontrados por Torre (2007), Villardón-Gallego et al. (2013) y Tesouro, Cañabate, \& Puiggalí (2014).

Si analizamos globalmente por curso a los estudiantes, solo se diferencian de forma estadísticamente significativa en el Uso del Enfoque Superficial, siendo los de últimos cursos los que perciben utilizar más este tipo de enfoque. Sin embargo, no hay diferencias al analizar el nivel de desarrollo de la competencia de aprender a aprender a medida que avanzan los cursos.

La utilidad de proponer instrumentos de evaluación de competencias, como es la competencia de aprender a aprender, se basa en hacer explícitas, para el profesorado, tanto universitario como de los niveles de Educación Secundaria y Bachillerato, las dimensiones e indicadores que definen la competencia, esto les permitirá diseñar y utilizar metodologías didácticas adecuadas para su desarrollo y evaluación de acuerdo a lo marcado en las programaciones. Estos aspectos, aunque muy integrados en los diseños de títulos, están muy poco desarrollados en las metodologías didácticas y de evaluación utilizadas por la dificultad de aplicación que ofrecen muchas de las propuestas realizadas, tanto por investigadores, como por formadores de profesorado.

Una de las decisiones didácticas fundamentales pasaría por alinear las estrategias de enseñanza que emplean los docentes con las diversas capacidades que integra la competencia de aprender a aprender y ambos elementos, a su vez, con los procedimientos de evaluación más adecuados para valorar el desarrollo de la competencia y poder potenciarlo.

Así por ejemplo, si nos centráramos en la autoevaluación del propio aprendizaje (aspecto que los estudiantes perciben tener menos desarrollado), cabría trabajar el componente hacer conscientes los pasos que han seguido en una actividad de aprendizaje para completarla con éxito. Modelar ejemplos concretos de la utilización de estrategias de aprendizaje que conducen al logro de determinados objetivos podría ser un buen modo de trabajar este aspecto con los estudiantes. Posteriormente, tras realizar una actividad de aprendizaje concreta, cabría analizar junto a ellos, mediante una rúbrica, si los pasos que han seguido han sido los más adecuados para alcanzar la meta de la actividad y en qué grado lo han hecho. Ésta sería una estrategia de evaluación coherente con la capacidad que estamos trabajando y con lo que se ha trabajado en el aula.

\section{Referencias}

Bandura, A. (1986). Social foundations of thought and action: A social cognitive theory. Englewood Cliffs, NJ: Prentice Hall.

Bandura, A. (1991). Social cognitive theory of self-regulation. Organizational Behavior and Human Decision Processes, 5, 248-287.

Bentler, P. M. (1995). EQS. Structural Equations Program Manual. Encino, CA: Multivariate Software.

Biggs, J., Kember, D., \& Leung, D. (1991). The revised two-factor Study Process Questionnaire: R-SPQ-2F. British Journal of Educational Psychology, 71(1), 133-149. 
Bolívar, A. (2009). Aprender a aprender a lo largo de la vida. Multiárea. Revista de Didáctica, 4, 63-96.

Broc-Cavero, M.A. (2011). Voluntad para estudiar, regulación del esfuerzo, gestión eficaz del tiempo y rendimiento académico en alumnos universitarios. Revista de Investigación Educativa, 29(1), 171-184. Recuperado de http://revistas.um.es/rie/article/ view/110731/126992

Carretero, R., \& Fuentes, M. (2010). La competencia de aprender a aprender. Aula de Innovación Educativa, 192, 7-10.

Comisión Europea (1995). White Paper on Education and Training. Teaching and Learning Towards the Learning Society. Bruselas: Comisión Europea.

Consejo Europeo (2000). Conclusiones de la presidencia, Consejo europeo de Lisboa 23 y 24 marzo 2000. Bruselas: Comisión Europea. Recuperado de http://www.minhap.gob. es/Documentacion/Publico/SGPEDC/Estrategia\%20de\%20Lisboa.pdf

Consejo Europeo (2001). Conclusiones de la presidencia del Consejo Europeo celebrado en Estocolmo los días 23 y 24 de marzo de 2001. Bruselas: Comisión Europea.

Consejo Europeo (2002). Conclusiones de la presidencia del Consejo Europeo celebrado en Barcelona los días 15 y 16 de marzo de 2002. Bruselas: Comisión Europea.

Council of Europe (1997). Key competencies for Europe. Report of the Symposium in Berne 27-30March 1996. Strasbourg: Council of Europe.

Dávila, N., García-Artiles, M.D., Pérez-Sánchez, J.Mª., \& Gómez-Déniz, E. (2015). Un modelo de regresión logística asimétrico que puede explicar la probabilidad de éxito en el rendimiento académico. Revista de Investigación Educativa, 33(1), 27-45. Recuperado de http://revistas.um.es/rie/article/view/178481/170771

Deakin-Crick. R, Broadfoot, P., \& Claxton, G. (2004). Developing an Effective Lifelong Learning Inventory: the ELLI Project. Assessment in Education, 11(3), 247 - 272.

Education Council (2006). Recommendation of the European Parliament and the Council of 18 December 2006 on key competencies for lifelong learning. Brussels. Official Journal of the European Union, L 394, 10-18.

Elshout-Mohr, M., Meijer, J., Oostdam, R., \& Van Gelderen, A. (2004). CCST: A Test for Cross-Curricular Skills. Amsterdam: SCO - Kohnstamm Institution, University of Amsterdam

García-Ros, R., \& Pérez-González, F. (2011). Validez predictiva e incremental de las habilidades de autorregulación sobre el éxito académico en la universidad. Revista de Psicodidáctica, 16(2), 231-250.

Gutiérrez-Braojos, C., Salmerón-Pérez, H., \& Muñoz-Cantero, J.M. (2014). Moderator Effect of Time Orientation Patterns in Achieving Self-Regulated Learning. Revista de Psicodidáctica, 19(2), 267-287.

Hautamäki, J., Arinen, P., Eronen, S., Hautamäki, A., Kupianien, S., Lindblom, B., Niemivirta, M., Pakaslahti, L., Rantanen, P., \& Scheinin, P. (2002). Assessing Learning-to-Learn: A Framework. Helsinki: Centre for Educational Assessment, Helsinki University / National Board of Education.

Hernández-Franco V., \& Gonzalo-Misol, I. (2009). Validación de un sistema de indicadores para valorar la integración socioeducativa de alumnos emigrantes. En B. Boza-Carreño, J.M. Méndez-Garrido, M. Monescillo-Palomo, M. Toscano-Cruz, M. Aguaded-Gómez, J.A. Ávila Fernández, M. Tello Díaz, \& M. Salas Tenorio (Coords.), 
Educación, investigación y desarrollo social. Actas del XIV Congreso Nacional de modelos de Investigación Educativa (AIDIPE) (239-280). Huelva: Universidad de Huelva.

Hoskins, B., \& U. Fredriksson (2008). Learning to Learn: What is it and can it be measured? JRC Scientific and Technical Report.

Hu, L., \& Bentler, P. M. (1999). Cutoff criteria for fit indexes in covariance structure analysis: conventional criteria versus new alternatives. Structural Equation Modeling, 6, 1-55.

Jornet-Meliá, J.M., García-Bellido, R., \& González-Such, J. (2012). Evaluar la competencia aprender a aprender: una propuesta metodológica. Revista de Currículum y formación del profesorado, 16(1). Recuperado de http://www.ugr.es/ recfpro/rev161ART7.pdf

Kupiainen, S., Hautamäki, J., \& Rantanen, P. (2008): EU pre-pilot on Learning to Learn. Report on the compiled data. University of Helsinki. Recuperado de http://ec.europa. eu/education/lifelong-learning-olicy/doc/pilot_survey/report_en.pdf

Leat, D., Thomas, U., \& Reid, A. J. (2012). The Epistemological Fog in Realising Learning to Learn in European Curriculum Policies. European Educational Research Journal, 11(3), 400-412.

López-Aguado, M. \& Gutiérrez-Provecho, L. (2014). Modelo explicativo del efecto de los enfoques de aprendizaje sobre el rendimiento y el papel modulador de la dedicación temporal. Revista de Investigación Educativa, 32(2), 447-462. Recuperado de http://revistas.um.es/rie/article/view/164761/163271

Martín, E., \& Moreno, A. (2007). Competencia para aprender a aprender. Madrid: Alianza Editorial.

Martínez-Fernández, J. R., García-Ravidá, L., González Velázquez, L., Gutiérrez-Braojos, C., Poggioli, L., Ramírez Otálvaro, P., \& Telleria, M. B. (2009). Inventario de los Estilos de Aprendizaje en Educación Superior. Versión en castellano del Inventory of Learning Styles-ILS. Barcelona: Universitat Autònoma de Barcelona.

Martínez-Fernández, R. (2007). Concepción de aprendizaje y estrategias metacognitivas en estudiantes universitarios de psicología. Anales de Psicología, 23(1), 7-16.

Meyer, D.K., \& Turner, J.C. (2002). Using instructional discourse analysis to study the scaffolding of student regulation. Educational Psychologist, 37(1), 17-25.

Moreno, A. (2002). La evaluación de las habilidades metacognitivas. En Marchesi, A. \& Martín, E. (Eds.), Evaluación de la educación secundaria. Madrid: Fundación Santa María, 119-136.

Moreno, A., Cercadillo, L., \& Martínez, M. (2008). Learn European Project. Pre-Pilot Study National Report. Spain. Instituto de Evaluación. Madrid: Ministerio de Educación.

Muñoz-San Roque, I., \& Martínez-Felipe, M. (2012). Enfoques de aprendizaje, expectativas de autoeficacia y autorregulación, ¿las metodologías de enseñanza utilizadas en el proyecto piloto del EEES en E2 afectan a la calidad del aprendizaje? En Muñoz San Roque, I. (Coord.), El Espacio Europeo de Educación Superior: ¿un cambio deseable para la Universidad? Madrid: Universidad Pontificia Comillas, 24-47.

Muñoz-San Roque, I., Prieto-Navarro, L., \& Torre-Puente, J.C. (2012). Enfoques de aprendizaje, autorregulación, autoeficacia, competencias y evaluación en estudiantes de Educación Infantil y Primaria. En Torre Puente, J.C. (Ed.), Educación y nuevas sociedades. Madrid: Universidad Pontificia Comillas, 237-266.

O'Neil, H.F., \& Abedi, J. (1996). Reliability and validity of a state metacognitive inventory: Potential for alternative assessment. Journal of Educational Research, 89(4), 234-245. 
Pekrun, R., Goetz, T., Titz, W., \& Perry, R.P. (2002). Academic Emotions in Students' Self-regulated learning and achievement: A program of qualitative and quantitative research. Educational Psychologist, 37(2), 91-105.

Pintrich, P.R., \& De Groot, E.V. (1990). Motivational and self-regulated learning components of classroom academic performance. Journal of Educational Psychology, 82(1), 33-40.

Pintrich, P.R., Smith, D.A., García, T., \& Mckeachie, W.J. (1991). A manual for the use of the Motivated Strategies for Learning Questionnaire (MSLQ). Ann Arbor: University of Michigan.

Salmerón, H., \& Gutiérrez, C. (2012). La competencia para aprender a aprender y el aprendizaje autorregulado. Posicionamientos Teóricos. Revista de Currículum y Formación del Profesorado, 16(1), 5-13. Recuperado de http://www.ugr.es/ recfpro/rev161ART1.pdf

Salmerón, H., Gutiérrez, C., Salmerón, P., \& Rodríguez, S. (2011). Metas de logro, estrategias de regulación y rendimiento académico en diferentes estudios universitarios. Revista de Investigación Educativa, 29(2), 467-486. Recuperado de http://revistas.um.es/ rie/article/view/116041/135371

Savalei, V., \& Bentler, P.M. (2006). Structural equation modeling. En Grover R. \& Vriens M. (Eds.), The handbook of marketing research: Uses, misuses, and future advances. Thousand Oaks CA: Sage, 330-364.

Tesouro, M., Cañabate, D., \& Puiggalí, J. (2014). Los enfoques de aprendizaje en estudiantes universitarios catalanes mediante el Approaches and Study Skills Inventory for Students (ASSIST). Revista de Investigación Educativa, 32(2), 479-498. Recuperado de http://revistas.um.es/rie/article/view/181981/163291

Torre Puente, J.C. (2007). Una triple alianza para un aprendizaje universitario de calidad. Madrid: Universidad Pontificia Comillas.

Unión Europea (2007). Competencias clave para el aprendizaje permanente: Un Marco de Referencia Europeo. Luxemburgo: Oficina de Publicaciones Oficiales de las Comunidades Europeas.

Valle, J., \& Manso, J. (2013). Competencias clave como tendencia de la política educativa supranacional de la Unión Europea. Revista de Educación, Extraordinario, 12-33.

Villardón-Gallego, L., Yániz, C. Achurra, C. Iraurgi, J., \& Aguilar, C. (2013). Learning competence in university: development and structural validation of a scale to measure. Revista de Psicodidáctica, 18(2), 357-374.

Zimmerman, B. J. (1989). A social cognitive view of self-regulated academic learning. Journal of Educational Psychology, 81, 329-339.

Zimmerman, B.J. (1990). Self-regulated learning and academic achievement: an overview. Educational Psychologist, 25(1), 3-17.

Zimmerman, B.J. (1995). Self-regulation involves more than metacognition: A social cognitive perspective. Educational Psychologist, 30(4). 217-221.

Zimmerman, B.J. (2013). From Cognitive Modeling to Self-Regulation: A Social Cognitive Career Path. Educational Psychologist, 48(3), 135-147.

Fecha de recepción: 25 de agosto de 2015.

Fecha de revisión: 25 de agosto de 2015.

Fecha de aceptación: 29 de marzo de 2016. 
